\title{
Limiting the stroke of a Schmitt trigger with multiplicative noise
}

\author{
Gabriel A. Zarza, Sergio E. Mangioni, Javier Fernandez Acevedo, and Roberto R. Deza* \\ IFIMAR (Universidad Nacional de Mar del Plata and CONICET), Deán Funes 3350, B7602AYL Mar del Plata, Argentina
}

(Received 1 December 2016; revised manuscript received 30 March 2017; published 25 May 2017)

\begin{abstract}
We have devised an experiment whereby a bistable system is confined away from its deterministic attractors by means of multiplicative noise. Together with previous numerical results, our experimental results validate the hypothesis that the higher the slope of the noise's multiplicative factor, the more it shifts the stationary states.
\end{abstract}

DOI: 10.1103/PhysRevE.95.052143

\section{INTRODUCTION}

Stochastic modeling is by now a usual methodology in almost every quantitative discipline. A grand subclass of these models follows the paradigm set forward more than a century ago by Langevin [1], who supplemented the Newtonian one by forcing the dynamic equations with random increments that obey certain statistics. The disposal of enough computing power triggered some 30 years ago the widespread application to the factual sciences of this methodology (well suited in fact to the increasing parallelism of computational architectures).

The role of fluctuations in the dynamics of lumped and extended [2-4] nonlinear deterministic systems has been from the outset a central topic in nonequilibrium statistical physics. They clearly decide the fate of the system at instabilities (examples being spontaneous symmetry breakdown and the simplest forms of pattern formation [5]), but even activation phenomena (nucleation, first-order phase transitions, and chemical and nuclear reactions [6]) are disabled in their absence (we refer to these multistability problems as noise-assisted phenomena).

Given the high diversity of the nonlinear world (beyond our efforts to cast bifurcations into normal forms), the repertoire of noise effects on nonlinear systems is also large. As a rule, the (nonrigorous albeit effective in simpler cases) mean-field approximation, whereby one replaces a fluctuating variable by its mean, gives wrong results in more complex cases. It has become customary to speak of constructive effects of the noise, a field which continues to amaze us with counterintuitive results after decades of intensive research.

The simplest instances of some of these noise-induced phenomena rely on properties of the nonlinear deterministic systems to which they apply (bifurcations and characteristic lengths in pattern formation, characteristic times in stochastic and coherence resonance [7], and explicit bias in noise-induced transport [8]), so they occur even with additive noise. Otherstypically, noise-induced transitions [9] and phase transitions (featuring ergodicity breakdown) [10-12]—usually need the system to be subjected to multiplicative noise in order to take place (first-order noise-induced phase transitions may occur under additive noise [13]). The multiplicative noise may result either from intrinsic fluctuation of some parameter or from coupling some function of the system's dynamical variables to external noise.

The subject of this work is akin to noise-induced transitions (and under the definition given in Ref. [14], it is to be

*smangio@mdp.edu.ar considered as such), but its motivation arose from the context of noise-induced phase transitions, which we refer to in the next paragraph. For the time being, recall that among the traits of multiplicative noises is their ability to compete with the deterministic forces (see Appendix A). The bid between forces of deterministic and stochastic origins can lead to a reorganization of the system (which is called constructive effects of the noise).

The first class of noise-induced phase transitions to be discovered was that of those driven by a short-time instability $[10,15,16]$, which curiously ruled out those systems displaying noise-induced transitions. This transition is reentrant with noise intensity and for colored noise it turns out to be also reentrant with the self-correlation time [17-20]. A second class was discovered not much later on, supported by the so-called entropic mechanism [11,12,21-23] (see Appendix B). Besides being compatible with noise-induced transitions, it provides a handy tool for stabilizing nanopatterns [24-26] — and inducing pinning states and dissipative solitons [27]—in adsorbed monolayers, as well as in models from ecology [28,29]. In all the cases, the following crucial hypothesis was explicitly used with positive results: "The noise's multiplicative factor $\Gamma^{1 / 2}(u)$ will affect more the solutions of $Q_{\text {eff }}(u)=0$ the higher its slope" [24-30] (see Appendix B for definitions).

A numerical test of this hypothesis in $d=0$ (as defined in the Appendix B) was performed in Ref. [31]. In a simple system whose deterministic dynamics is governed by a cubic normal form, a multiplicative noise was introduced with the specific purpose of confining the system between prefixed bounds, away from its deterministic attractors. According to the hypothesis, the expectation was that a noise intense enough would drive the system toward the region where the multiplicative factor (and hence the noise) is minimal.

In accordance with our previous results [24-29,31], we interpret that the possible stationary solutions are defined by a bid between deterministic and stochastic forces. The former push the system toward their attractors (their stable zeros) and the latter push it toward the minima of the multiplicative factor. In the numerical simulations [31], the stationary solutions for moderate noise intensities were located within the confinement region but their distance to the multiplicative factor's minima was still appreciable. However, as the noise intensity continued to increase, they became coincident for all practical purposes.

In the following we describe an electronic circuit devised to recreate the situation considered in Ref. [31] and report experimental results supporting the hypothesis. A Schmitt trigger is used as a metaphor for the cubic normal form, and external Gaussian wideband noise from an analog source is 
multiplicatively incorporated through a specifically designed circuit. In the following section we introduce the model, find its analytical stationary probability density function (SPDF), and compare it with the one found in simulations [31]. In the next sections we describe the experimental setup and results, and summarize our conclusions.

\section{MODEL AND PREVIOUS RESULTS}

The model considered in Ref. [31] is described by

$$
\dot{u}=Q(u)+\Gamma^{1 / 2}(u) \eta(t),
$$

with $Q(u)=u\left(1-u^{2}\right)$ and $\left\langle\eta(t) \eta\left(t^{\prime}\right)\right\rangle=2 \lambda^{2} \delta\left(t-t^{\prime}\right)$. It is our purpose to confine its stationary states away from the deterministic solutions $u= \pm 1$ (i.e., between $u= \pm u_{l}$, with $u_{l}<1$ ) using a suitable multiplicative noise. In order to achieve that goal, we propose

$$
\begin{aligned}
\Gamma^{1 / 2}(u) & :=1-\Theta\left(u_{l}-|u|\right)\left\{1-\exp \left[b\left(|u|-u_{l}\right)\right]\right\} \\
& =\left\{\begin{array}{lll}
\exp \left[b\left(|u|-u_{l}\right)\right] & \text { if } \quad|u|<u_{l} \\
1 & \text { if } & |u|>u_{l} .
\end{array}\right.
\end{aligned}
$$

Thus the effect of the noise decreases exponentially within the confinement region and is maintained at its maximum intensity level in the forbidden region [32]. For $Q(u)=u\left(1-u^{2}\right), u \leftrightarrow$ $-u$ symmetry is expected.

The SPDF can be calculated using either Eq. (A5) (with $\alpha=1 / 2$, corresponding to Stratonovich's interpretation) or Eq. (B2). For $|u|<u_{l}$ we obtain

$\mathcal{N} P_{\mathrm{th}}^{<}(u)=\exp \left\{-\frac{e^{2 b u_{l}}}{4 b^{2} \lambda^{2}}\left[H(u)-1+\frac{3}{2 b^{2}}\right]-b\left(|u|-u_{l}\right)\right\}$

$\left(\mathcal{N}\right.$ is the normalization factor), with $H(u)=S(u) e^{-2 b|u|}$ and

$$
S(u)=\left[2 b|u|+1-\frac{4 b^{3}|u|^{3}+6 b^{2}|u|^{2}+6 b|u|+3}{2 b^{2}}\right],
$$

whereas for $|u|>u_{l}$,

$$
P_{\mathrm{th}}^{>}(u)=P_{\mathrm{th}}^{<}\left(u_{l}\right) \exp \left[\frac{u^{2}-u_{l}^{2}-\left(u^{4}-u_{l}^{4}\right) / 2}{2 \lambda^{2}}\right] .
$$

Figure 1 displays $P_{\text {th }}(u)$ in Eqs. (3) and (4), for $\lambda=5$ and $b=20$ (inside the bounds: thin line). As seen, $P_{\text {th }}^{>}(u)$ in Eq. (4)-lying outside the vertical lines that indicate the boundaries of the confinement region-is vanishingly small. This is in accordance with the numerically calculated SPDF [33] (inside the bounds: thick line). For additive noise $(\lambda=$ $\sqrt{.02}$ ) instead, the SPDF has its maxima outside the bounds and is vanishingly small inside [34].

In Ref. [31], Eq. (1) was numerically integrated using a Heun scheme with time step $\Delta t=10^{-5}$ (results not shown here). The confinement is seen to improve as $\lambda$ increases. Moreover, past some threshold value of $\lambda$, the curves display transitions between the two possible solutions localized within the region of confinement. If the system starts with $u<0$, the

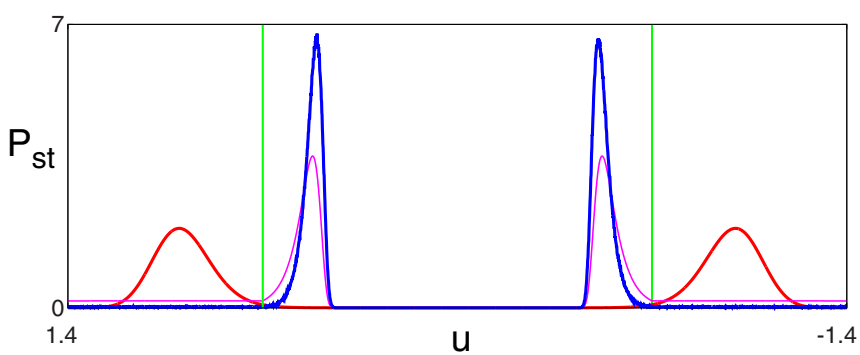

FIG. 1. Shape of the SPDF $P_{\text {st }}(u)$ in three instances. Constant- $u$ lines indicate the boundaries of the confinement region $u_{l}= \pm 0.7$. For additive noise $(\lambda=\sqrt{.02})$, the SPDF has its maxima outside the bounds. For $\lambda=5$ and $b=20$ instead, it becomes confined (inside the bounds: thin line, analytical calculation; thick line, numerical calculation) [31].

curves are the mirror image of the ones obtained when it starts with $u>0$.

\section{EXPERIMENTAL SETUP}

It is clear from Eq. (1) that an experimental setup to test the validity of our proposal will consist of three main blocks: the function $Q(u)$, the function $\Gamma^{1 / 2}(u)$, and the noise $\eta(t)$. However, the global structure of the circuit is better appreciated by recasting Eq. (1) in a form that resembles the OrnsteinUhlenbeck equation, namely,

$$
\tau \dot{u}=-u+F(t ; u),
$$

with $F(t ; u):=u+Q_{e}(u)+\Gamma_{e}^{1 / 2}(u) \eta(t)$ [35]. Equation (5) highlights the fact that $\tau$ governs the system's dynamics around the attractors for $\lambda \rightarrow 0$ and, hence, it determines the cutoff frequency in the spectral density of $u$. In the following, the state-dependent noise $F(t ; u)$ is denoted simply as $F(u)$. Moreover, $Q_{e}(u)$ and $\Gamma_{e}^{1 / 2}(u)$ denote the experimental counterparts of $\tau Q(u)$ and $\tau \Gamma^{1 / 2}(u)$.

Figure 2 shows a schematic block diagram of the devised electronic circuit. Besides the obvious passive components, the main components are AD826 operational amplifiers. Referring now to Eq. (5), the crucial element that organizes the picture in Fig. 2 is the adder circuit, which implements the noise $F(u)$. It is represented by a circle containing three plus signs, toward which three arrows converge. The upper arrow inputs $Q_{e}(u)$, the one at the left inputs the variable $u$ under study, and the lower one inputs $\Gamma_{e}^{1 / 2}(u)$. The remaining block is just a resistor-capacitor (RC) filter (clearly, $\tau=R C$ ) - submitted to the noise source $F(u)$ as described by Eq. (5)—whose output is plotted in Figs. 3, 5, and 6.

Function $Q_{e}(u)$. The function $Q_{e}(u)$ is emulated by a Schmitt trigger (a positive-feedback operational amplifier with decision thresholds), so designed that its output voltage is centered around $0 \mathrm{~V}$, it varies across $\pm 10 \mathrm{~V}$, and its stationary solutions are $\pm u^{s}= \pm 5 \mathrm{~V}$ [36].

Figure 3 shows the output $u$ of the RC filter (the input voltage of the Schmitt trigger) in the absence of multiplicative noise (i.e., $\lambda=0$ ). Internal electronic noise causes transitions over $\pm u^{s}$. The apparent regularity of the output is not due to an external regular signal, but to the fact that switchings due to fluctuations have a characteristic frequency (the inverse of 


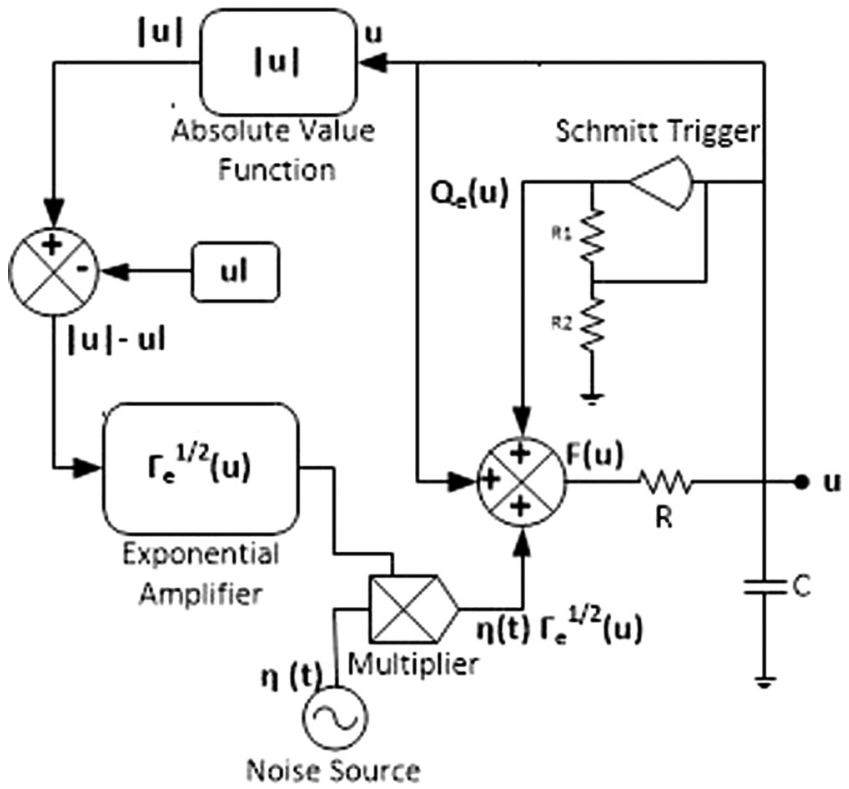

FIG. 2. Schematic block diagram of the electronic device. $u$ is the output voltage of the feedback system; the noise's multiplicative factor $\Gamma_{e}^{1 / 2}(u)$ is the input-output function of the exponential amplifier; $Q_{e}(u)$ is the input-output function of the Schmitt trigger, which mimics the cubic nonlinearity; $\eta(t)$ is the external Gaussian noise; and $F(u)=u+Q_{e}(u)+\Gamma_{e}^{1 / 2}(u) \eta(t)$.

the mean lifetime between transitions) [37], and the circuit's time constant $\tau$ is large enough to filter out higher frequencies that would make the response look more irregular.

Function $\Gamma_{e}^{1 / 2}(u)$. Referring to Eq. (2), the operations to be performed on $u$ are (i) taking its absolute value by means of a precision rectifier, (ii) comparing the latter with the prefixed bound $u_{l}$ (again, an adder), and (iii) exponentiating

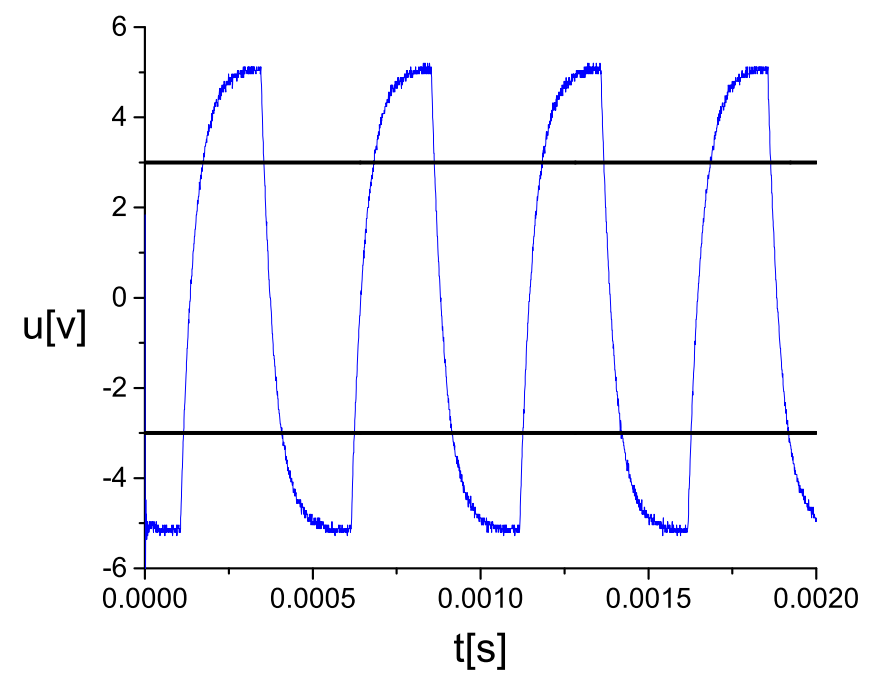

FIG. 3. Time window on the 2-ms scale of $u(t)$-the system's output voltage and Schmitt trigger's feedback input-without multiplicative noise $(\lambda=0 \mathrm{~V})$. The transitions are due to the internal (additive) electronic noise, which is always present in these devices. Constant $-u$ lines indicate the boundaries of a confinement region for $u_{l}= \pm 3 \mathrm{~V}$.
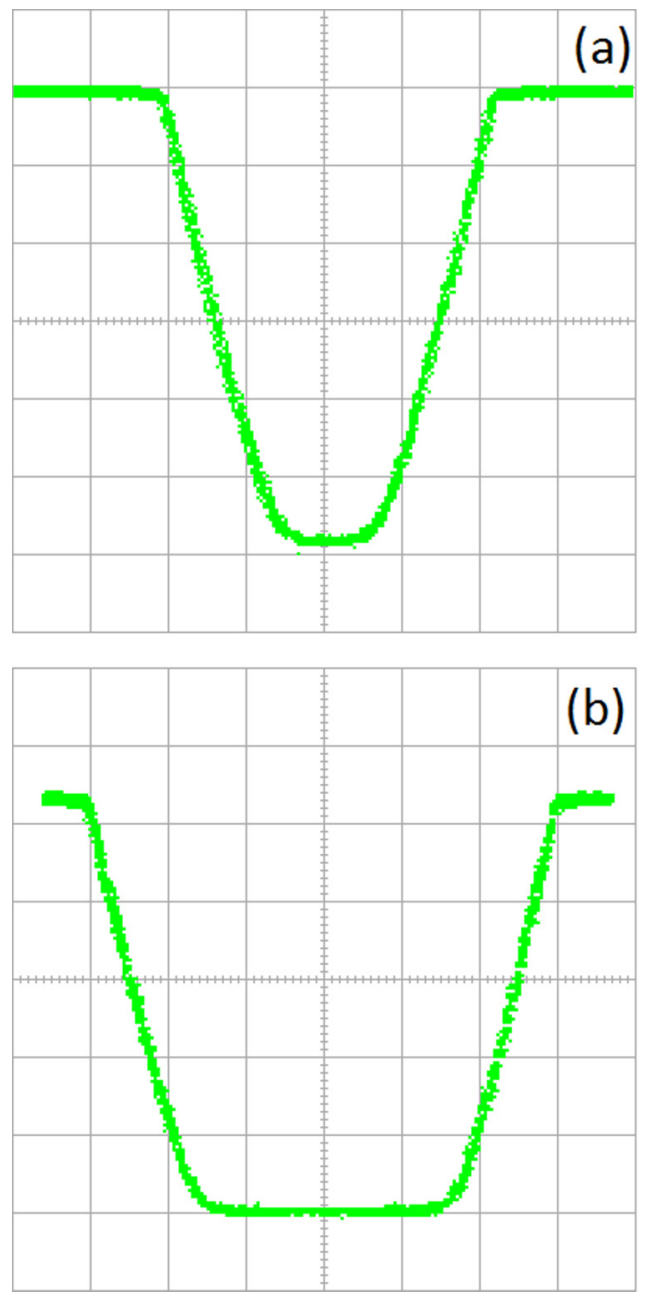

FIG. 4. Scope screen shots of the electronically synthesized input-output function $\Gamma_{e}^{1 / 2}(u)$ of the exponential amplifier, for (a) $u_{l}= \pm 2 \mathrm{~V}$ and (b) $u_{l}= \pm 3 \mathrm{~V}$. Oscilloscope use mode, $\mathrm{XY}$; channel A, $1.00 \mathrm{~V}$; channel B, $2.00 \mathrm{~V}$; and sampling frequency, $1 \mathrm{Ms} / \mathrm{s}$.

the result by means of an exponential amplifier, which uses the characteristic $I_{d}-V_{d}$ curve of a $1 \mathrm{~N} 4148$ diode [38].

Figure 4 shows scope screen shots of the input-output function of the exponential amplifier $\Gamma_{e}^{1 / 2}(u)$, for $u_{l}= \pm 2$ and $\pm 3 \mathrm{~V}$. The curves can be reinterpreted as dips in noise intensity as a function of $u$, designed to confine the system [i.e., to force $u(t)$ to remain inside the well].

Noise $\eta(t)$. Gaussian noise-generated by an analog source and filtered by a sixth-order Sallen-Key filter with $-3 \mathrm{~dB}$ cutoff frequency at $10 \mathrm{kHz}$-is injected together with $\Gamma_{e}^{1 / 2}(u)$ into an analog multiplier in the device. So even though it is colored (its spectral density decays as $f^{-6}$ ) for $f>10 \mathrm{kHz}$, it can be safely regarded as white at the working frequencies (around $1 \mathrm{kHz}$ ).

\section{EXPERIMENTAL RESULTS}

In order to test the effectiveness of the mechanism, we have recorded the time evolution of $u$ for different noise intensities, using an oscilloscope (Hantek DSO5062B). 


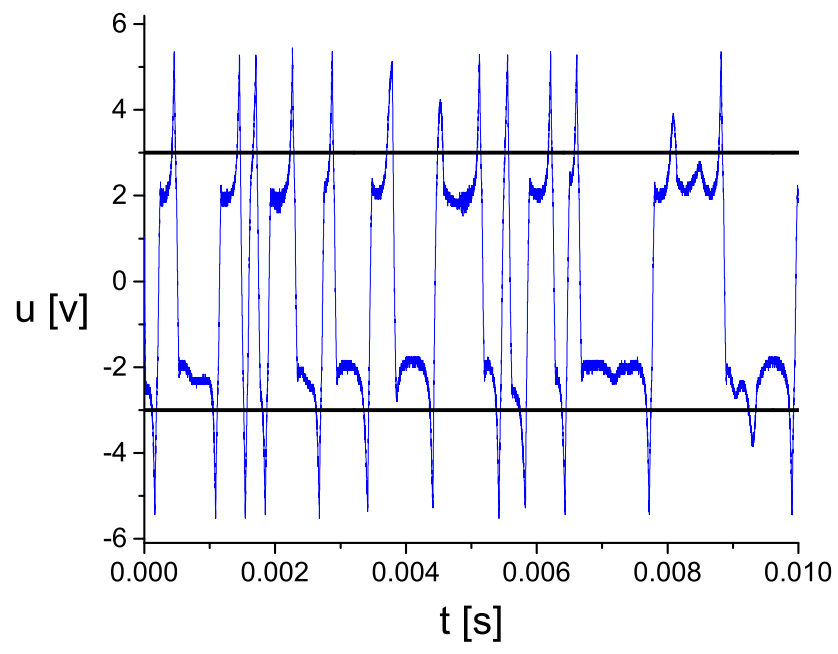

FIG. 5. Time window on the 10 -ms scale of the output voltage $u(t)$ for $u_{l}= \pm 3 \mathrm{~V}$ (constant $-u$ lines) and $\lambda=3.18 \mathrm{~V}$, illustrating switchings between two basically stationary solutions that lie inside the confinement region. The detailed dynamics of the Schmitt trigger is such that internal (additive) electronic noise succeeds in driving the system outside the confinement region during very short periods before each switching.

Figure 5 shows - on a somewhat longer time scale than Fig. $3-u$ versus $t$ for $\lambda=3.18 \mathrm{~V}$ and $u_{l}= \pm 3 \mathrm{~V}$. Even though each noise-driven switch is preceded by a short transient pulse taking the system outside the prefixed bounds, the system remains confined on time average (and also in probability, as shown in Fig. 7). We conjecture that what these "runoffs" are telling us is that the Schmitt trigger is not a totally faithful representation of $Q_{e}(u)$.

Figure 6 is a close-up of time series like the one in Fig. 5, focusing on periods during which the system remains near $+u^{s}(\lambda)$; i.e., neither the transitions nor the runoffs are seen. The values of $u_{l}$ and $\lambda$ are listed in Table I. In particular, the lowest-lying curve in the lower frame has been extracted from the same time series as Fig. 5.

Since the system is subjected all the time to both the external multiplicative noise and the internal additive noise, the mean lifetime between transitions depends in principle on both. From Fig. 6, one can conclude that the former is pretty effective in maintaining the system near $+u^{s}(\lambda)$, so clearly the lifetime is basically determined by the latter. However, since apparently the system's response to internal noise is in the form of very short runoff pulses, it is not expected to contribute largely to the SPDF.

Figure 7 completes the picture by showing the experimental SPDF for the cases in Fig. 6. Starting from a stationary state corresponding to given $\lambda$ and $u_{l}$, we recorded five long (1 000000 samples each, at a sampling frequency of $1.5 \mathrm{Ms} / \mathrm{s}$ ) time series of $u(t)$. For each series, we calculated its SPDF by dividing the variability range of $u$ into 150 cells and counting the number of times that $u(t)$ entered each cell. (N.B.: The data corresponding to undesired short pulses were not filtered.) After that, we averaged together the five SPDFs. The qualitative similarity of Fig. 7 with Fig. 1 is evident (the maximum in $P_{\text {st }}$ is displaced toward confinement region).

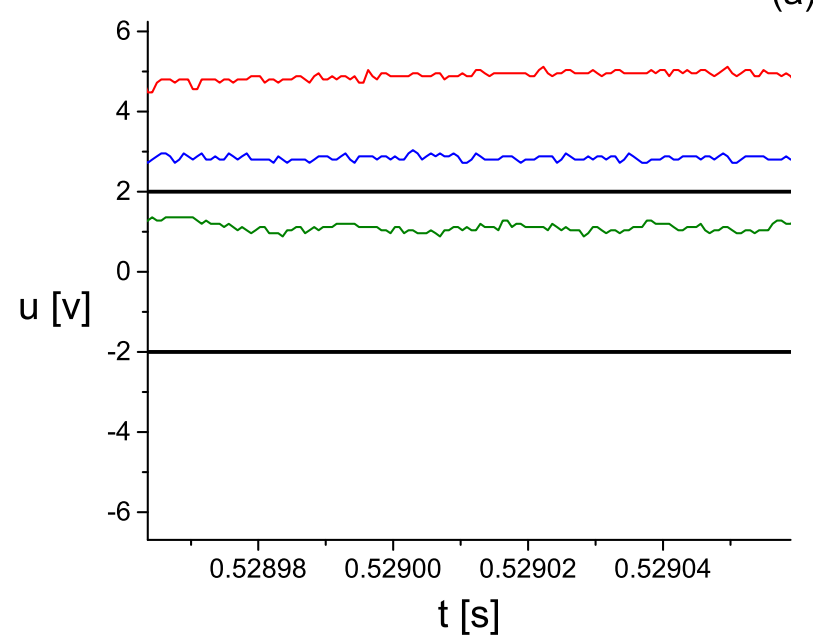

(a)

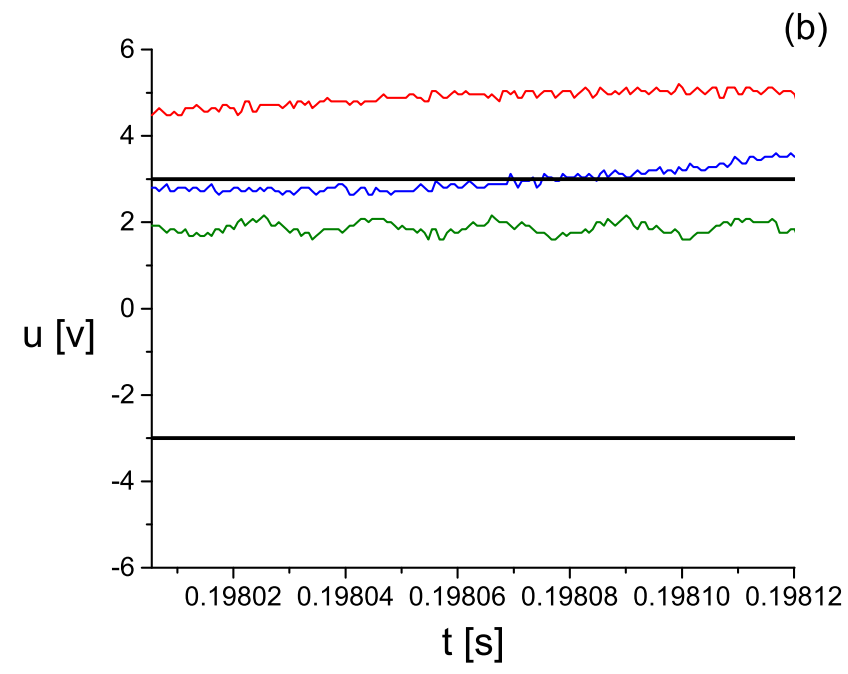

FIG. 6. Time windows on the 1-ms scale of the output voltage $u(t)$ between transitions, for the values of $u_{l}$ and $\lambda$ in Table I: (a) $u_{l}= \pm 2 \mathrm{~V}$ and (b) $u_{l}= \pm 3 \mathrm{~V}$. As $\lambda$ increases, the statistically stationary (before runoff) states shift into the confinement region.

\section{CONCLUSIONS}

The fact that a multiplicative noise drives the system toward a state that minimizes its multiplicative factor (if this is positive) can be taken as a principle, and has theoretical and practical implications. On the one hand, it allows one to predict the outcome of a given situation just by looking at the noise multiplicative factor. On the other hand, it is possible to devise stochastic environments (e.g., by means of computercontrolled masks in a photosensitive chemical reaction-at least down to the pixel scale) that promote stationary states other than the deterministic ones. As measured by its effects,

TABLE I. Parameters in Figs. 6 and 7.

\begin{tabular}{lcccc}
\hline \hline Frame & $u_{l}(\mathrm{~V})$ & \multicolumn{3}{c}{$\lambda(\mathrm{V})$} \\
\hline (a) & 2.00 & 0.00 (top) & 0.54 (middle) & 3.17 (bottom) \\
(b) & 3.00 & 0.00 (top) & 0.44 (middle) & 3.18 (bottom) \\
\hline \hline
\end{tabular}


(a)

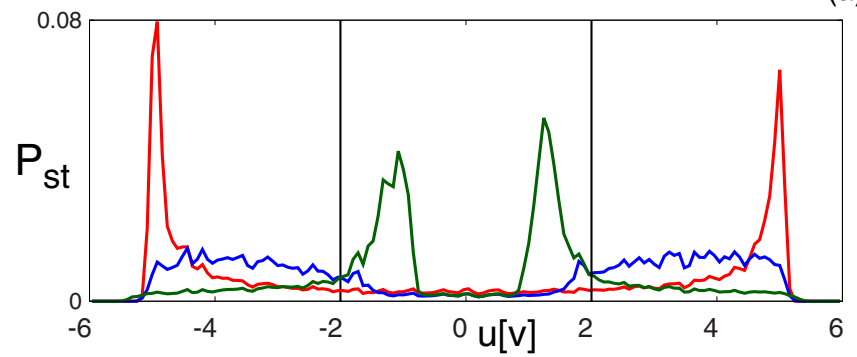

(b)

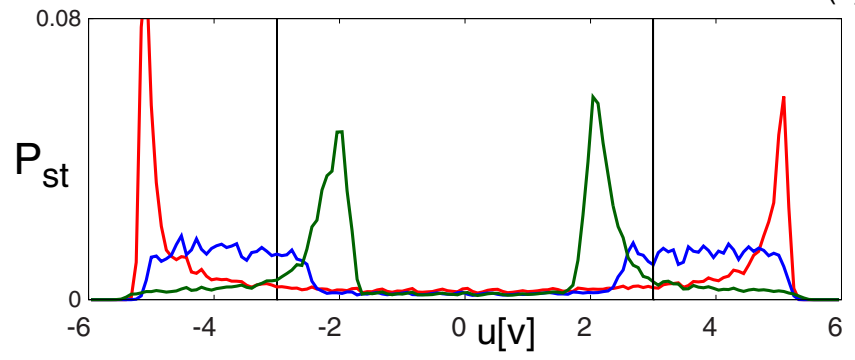

FIG. 7. Experimental SPDF for the cases in Fig. 6. Constant- $u$ lines indicate the boundaries of the confinement region: (a) $u_{l}= \pm 2 \mathrm{~V}$ and (b) $u_{l}= \pm 3 \mathrm{~V}$. As $\lambda$ increases, the experimental SPDF changes from peaking outside to peaking inside the confinement region.

the stochastic force-tending to minimize the effect of the multiplicative noise and driven by the negative gradient of its factor-might even be considered at an equal foot with the a priori deterministic forces [39]. As mentioned in the introduction, the hypothesis under test in this work has given rise to several theoretical investigations. But until now, no experiment corroborating this remarkable effect of the multiplicative noise had been carried out. To our judgment, the experiment described in this work gives full support to the hypothesis stated in the introduction. Moreover, it is a robust effect, as can be inferred from the fact that the Gaussian noise used in our experiment is white only up to $10 \mathrm{kHz}$.

It is also worth remarking that this experiment is not an analog simulation but a real physical setup. The noise is ultimately produced by physical phenomena inside electronic devices, and the used components can be regarded as concrete examples of the abstract class of physical systems under investigation. Incidentally, we have recently come to know about an interesting experiment on noise-induced transitions in a double-well oscillator with nonlinear dissipation (clearly differing from ours in their objectives), of which the same can be said [40].

\section{ACKNOWLEDGMENT}

Financial support from CONICET and UNMdP of Argentina is acknowledged.

\section{APPENDIX A: EFFECT OF THE MULTIPLICATIVE NOISE}

We consider first $n=1$ dynamical variables. The (conditional) probability density function (PDF) $P\left(x, t \mid x_{0}, 0\right)$ of a Markov process $x(t)$ that obeys the generalized Langevin equation

$$
\dot{x}=f(x)+g(x) \eta(t),
$$

$\eta$ being a Gaussian white noise with $\left\langle\eta(t) \eta\left(t^{\prime}\right)\right\rangle=2 \lambda^{2} \delta\left(t-t^{\prime}\right)$, obeys in turn the Fokker-Planck equation (FPE)

$$
\partial_{t} P\left(x, t \mid x_{0}, 0\right)+\partial_{x} J\left(x, t \mid x_{0}, 0\right)=0 .
$$

In terms of the Kramers-Moyal coefficients [41],

$$
J\left(x, t \mid x_{0}, 0\right)=D^{(1)}(x) P-\partial_{x}\left[D^{(2)}(x) P\right] .
$$

Whereas the diffusion coefficient $D^{(2)}(x)=\lambda^{2} g^{2}(x)$ is well determined, the drift one $D^{(1)}(x)$ involves the integral

$$
I(t, d t):=\int_{t}^{t+\mathrm{d} t} g(x) \eta(u) d u
$$

which, at variance with

$$
\int_{t}^{t+\mathrm{d} t} \eta(u) d u=d W(t):=W(t+d t)-W(t)
$$

which holds uniquely ( $W$ being the Wiener process), must be defined or interpreted.

Stratonovich regarded the highly singular white noise as the limit of a physical noise with very large bandwidth. His definition

$$
I(t, d t):=g\left(\frac{x(t)+x(t+d t)}{2}\right) d W(t)
$$

preserves the chain rule of calculus, but $D^{(1)}$ picks up a contribution from the noise term:

$$
D^{(1)}=f(x)+\lambda^{2} g(x) g^{\prime}(x)
$$

(the prime indicates total derivative). Itô's definition $I(t, d t):=$ $g(x(t)) d W(t)$ (popular among mathematicians and arising from handling the $\delta$ function otherwise) yields indeed $D^{(1)}=$ $f(x)$, but at the cost of changing the rules of calculus.

Even though we attach to Stratonovich's interpretation, we first show that the underlying phenomenon has little to do with the interpretation of $I(t, d t)$. After the immediate generalization

$$
I(t, d t):=g([1-\alpha] x(t)+\alpha x(t+d t)) d W(t),
$$

$J\left(x, t \mid x_{0}, 0\right)$ in Eq. (A2) can be written as

$$
\begin{aligned}
J\left(x, t \mid x_{0}, 0\right)= & {\left[f(x)-2 \lambda^{2}(1-\alpha) g(x) g^{\prime}(x)\right] P\left(x, t \mid x_{0}, 0\right) } \\
& -\lambda^{2} g^{2}(x) \partial_{x} P\left(x, t \mid x_{0}, 0\right) .
\end{aligned}
$$

If the FPE admits a SPDF $P_{\mathrm{st}}(x)$ such that $\partial_{t} P_{\mathrm{st}}(x)=0$, then $J_{\mathrm{st}}(x)=$ const, where $J_{\mathrm{st}}(x)$ in Eq. (A3) is evaluated for $P_{\mathrm{st}}(x)$. For natural boundary conditions, it is

$$
\left[\ln P_{\mathrm{st}}(x)\right]^{\prime}=\frac{1}{\lambda^{2} g^{2}(x)}\left[f(x)-2 \lambda^{2}(1-\alpha) g(x) g^{\prime}(x)\right],
$$

which, if $g>0$, can also be written as

$$
\left[\ln P_{\mathrm{st}}(x)\right]^{\prime}=\lambda^{-2} \frac{f(x)}{g^{2}(x)}-2(1-\alpha)[\ln g(x)]^{\prime} .
$$

Isolating the second term on the right-hand side of Eq. (A4) and regarding it as a dynamical flow we see that if $g>0$, its attractors are the minima of $g$. Hence, that term drives (pushes) the system to a state in which the effect of multiplicative noise 
is minimized, much the same way as the attractors of $f(x)$ are the minima of $V(x)=-\int_{0}^{x} f(y) d y$. Clearly, the stationary state in the presence of multiplicative noise will not be at the attractors of $f$, but will result of a bid between both terms. This may have dramatic consequences depending on the case, as, e.g., noise-induced transitions.

For $n>1$ it is $\dot{\mathbf{x}}=\mathbf{f}(\mathbf{x})+G(\mathbf{x}) \mathrm{H}(t) ; \operatorname{dim} \mathrm{H}=n$ and $\left\langle\mathrm{H}(t) \mathrm{H}\left(t^{\prime}\right)\right\rangle=2 I D \delta\left(t-t^{\prime}\right)$, but rank $G$ need not be $n$. The FPE is

$$
\partial_{t} P\left(\mathbf{x}, t \mid \mathbf{x}_{0}, 0\right)+\nabla \cdot \mathbf{J}\left(\mathbf{x}, t \mid \mathbf{x}_{0}, 0\right)=0,
$$

and the Kramers-Moyal coefficients become

$$
\begin{gathered}
D_{i}^{(1)}(\mathbf{x})=f_{i}(\mathbf{x})+2 \lambda^{2} \alpha G_{k j}(\mathbf{x}) \partial_{k} G_{i j}(\mathbf{x}), \\
\text { i.e., } D^{(1)}=\mathbf{f}+2 \lambda^{2} \alpha \hat{L}(\mathbf{x}) G^{T} \text { with } \hat{L}(\mathbf{x}):=\left(G^{T} \nabla\right)^{T} \text {, and } \\
D_{i j}^{(2)}(\mathbf{x})=\lambda^{2} G_{i k}(\mathbf{x}) G_{j k}(\mathbf{x}) \text { or } D^{(2)}=\lambda^{2} G G^{T},
\end{gathered}
$$

so $D^{(2)}$ is symmetric, rank $D^{(2)}=n$, and $\operatorname{det} D^{(2)}>0$. Equation (A4) reads

$\left[\ln P_{\mathrm{st}}(\mathbf{x})\right]^{\prime}=\left[D^{(2)}\right]^{-1}(\mathbf{x})\left[f_{i}(\mathbf{x})-2 \lambda^{2}(1-\alpha) G_{j k}(\mathbf{x}) \partial_{k} G_{i j}(\mathbf{x})\right]$,

so the conclusions remain valid. Of course for $n=1$ and $g>0$, we might have rewritten Eq. (A1) in terms of $y:=\int_{0}^{x} d x^{\prime} / g\left(x^{\prime}\right)$ and $h(y):=f[x(y)] / g[x(y)]$, to read $\dot{y}=$ $h(x)+\eta(t)$. Even though the attractors of $h$ will generically differ from those of $f$, noise-induced transitions occurring in terms of $x$ may not show up in terms of $y$ [14].

\section{APPENDIX B: THE ENTROPIC MECHANISM}

Originally, the entropic mechanism was introduced by proposing, for a field $u(\mathbf{x}, t)$, a relaxational flow with fielddependent kinetic coefficient $\Gamma(u)$ in a free energy $\mathcal{F}[u]$, and a noise term that fulfills the fluctuation-dissipation relation $[11,12]$. The fluctuating field $u(\mathbf{x}, t)$ obeys the stochastic partial differential equation

$$
\partial_{t} u(\mathbf{x}, t)=-\Gamma(u) \frac{\delta \mathcal{F}[u]}{\delta u(\mathbf{x}, t)}+\Gamma^{1 / 2}(u) \eta(\mathbf{x}, t),
$$

where $\eta(\mathbf{x}, t)$ is Gaussian, with $\langle\eta(\mathbf{x}, t)\rangle=0$ and

$$
\left\langle\eta(\mathbf{x}, t) \eta\left(\mathbf{x}^{\prime}, t^{\prime}\right)\right\rangle=2 \lambda^{2} \delta^{d}\left(\mathbf{x}-\mathbf{x}^{\prime}\right) \delta\left(t-t^{\prime}\right) .
$$

The virtue of this proposal is that it offers an analytic way of finding the SPDF for fields [11,12], namely,

$$
P_{\text {st }}[u] \propto \exp \left\{-\frac{\mathcal{F}_{\text {eff }}[u]}{\lambda^{2}}\right\},
$$

with an effective potential functional (hereafter, we attach to Stratonovich's interpretation)

$$
\mathcal{F}_{\text {eff }}[u]=\mathcal{F}[u]+\beta \int_{R} d x \ln \Gamma(u(x))
$$

(with $\beta \propto \lambda^{2}$ ) for a finite region $R$. If $R$ is made into a $d$-dimensional square lattice with mesh size $\Delta x$, then $\beta=$ $\lambda^{2} /\left(2 \Delta x^{d}\right)[11,12]$.

This idea can be extended to gradient flows (as, e.g., reaction-diffusion equations) by directly regarding $\Gamma(u)$ as the square of the noise multiplicative factor [24-29,31]. For simplicity, we restrict the discussion to $d=1$. Given

$$
\partial_{t} u=Q(u)+\partial_{x}\left[D(u) \partial_{x} u\right]+\Gamma^{1 / 2}(u) \eta(x, t),
$$

with $Q(u)$ being the reaction term and $D(u)$ a field-dependent diffusion coefficient, we define the fictitious functional $\mathcal{F}[u]$ from

$$
\frac{\delta \mathcal{F}[u]}{\delta u}:=-\frac{Q(u)+\partial_{x}\left[D(u) \partial_{x} u\right]}{\Gamma(u)} .
$$

We need not know $\mathcal{F}[u]$, but only assume it exists. Then by calculating $\delta \mathcal{F}_{\text {eff }}(u) / \delta u(x)$, we can describe the average effect of the noise on the deterministic dynamics given by $Q(u)+$ $\partial_{x}\left[D(u) \partial_{x} u\right]$. The equation governing the average dynamics in the presence of noise can be written as

$$
\partial_{t} u=Q_{\text {eff }}(u)+\partial_{x}\left[D(u) \partial_{x} u\right] .
$$

The effective reaction term $Q_{\mathrm{eff}}(u):=Q(u)-\beta \Gamma^{\prime}(u)$ allows us to observe the average effect of the noise on the homogeneous stationary states (HSS). It is quite clear that the higher the slope of the noise multiplicative factor, the more the solutions of $Q_{\text {eff }}(u)=0$ will change with the noise; recall the interpretation of the noise effect as a force displacing the HSS toward values that minimize $\Gamma(u)$. Then it would be possible to devise-or discover by eliciting the parameters' fluctuations [28,29]) - a noise multiplicative factor able to change the deterministic results completely, giving rise to more complex solutions.
[1] P. Langevin, C. R. Acad. Sci. (Paris) 146, 530 (1908).

[2] J. García-Ojalvo and J. M. Sancho, Noise in Spatially Extended System (Springer-Verlag, New York, 1999).

[3] F. Sagués, J. M. Sancho, and J. García-Ojalvo, Rev. Mod. Phys. 79, 829 (2007).

[4] H. S. Wio, R. R. Deza, and J. M. López, An Introduction to Stochastic Processes and Nonequilibrium Statistical Physics, revised ed. (World Scientific, Singapore, 2012).

[5] M. Cross and H. Greenside, Pattern Formation and Dynamics in Nonequilibrium Systems (Cambridge University Press, Cambridge, UK, 2009).

[6] P. Hänggi, P. Talkner, and M. Borkovec, Rev. Mod. Phys. 62, 251 (1990).
[7] L. Gammaitoni, P. Hänggi, P. Jung, and F. Marchesoni, Rev. Mod. Phys. 70, 223 (1998).

[8] P. Reimann, Phys. Rep. 290, 149 (1997).

[9] W. Horsthemke and R. Lefever, Noise Induced Transitions (Springer, Berlin, 1984).

[10] C. Van den Broeck, J. M. R. Parrondo, and R. Toral, Phys. Rev. Lett. 73, 3395 (1994).

[11] M. Ibañes, J. García-Ojalvo, R. Toral, and J. M. Sancho, Phys. Rev. Lett. 87, 020601 (2001).

[12] O. Carrillo, M. Ibañes, J. García-Ojalvo, J. Casademunt, and J. M. Sancho, Phys. Rev. E 67, 046110 (2003).

[13] A. A. Zaikin, J. García-Ojalvo, and L. Schimansky-Geier, Phys. Rev. E 60, R6275 (1999). 
[14] R. Toral, AIP Conf. Proc. 1332, 145 (2011).

[15] C. Van den Broeck, J. M. R. Parrondo, R. Toral, and R. Kawai, Phys. Rev. E 55, 4084 (1997).

[16] P. Reimann, R. Kawai, C. Van den Broeck, and P. Hänggi, Europhys. Lett. 45, 545 (1999).

[17] S. E. Mangioni, R. R. Deza, H. S. Wio, and R. Toral, Phys. Rev. Lett. 79, 2389 (1997).

[18] S. E. Mangioni, R. R. Deza, R. Toral, and H. S. Wio, Phys. Rev. E 61, 223 (2000).

[19] S. E. Mangioni, R. R. Deza, and H. S. Wio, Phys. Rev. E 63, 041115 (2001).

[20] S. E. Mangioni, R. R. Deza, and H. S. Wio, Phys. Rev. E 66, 051106 (2002).

[21] J. Buceta, M. Ibañes, J. M. Sancho, and K. Lindenberg, Phys. Rev. E 67, 021113 (2003).

[22] S. E. Mangioni and H. S. Wio, Phys. Rev. E 71, 056203 (2005).

[23] B. von Haeften, G. G. Izús, S. E. Mangioni, A. D. Sánchez, and H. S. Wio, Phys. Rev. E 69, 021107 (2004).

[24] S. E. Mangioni, Physica A 389, 1799 (2010).

[25] S. E. Mangioni and R. R. Deza, Phys. Rev. E 82, 042101 (2010).

[26] S. E. Mangioni and R. R. Deza, Physica A 391, 4191 (2012).

[27] S. E. Mangioni and R. R. Deza, Phys. Rev. E 92, 032116 (2015).

[28] S. E. Mangioni, Eur. Phys. J. B 88, 265 (2015).

[29] S. E. Mangioni, Phys. Rev. E 92, 062136 (2015).

[30] Analytical and numeric results illustrating this effect are shown in all these papers.

[31] S. E. Mangioni, Eur. Phys. J. B 88, 53 (2015).

[32] Strictly speaking, the condition $\Theta\left(u_{l}-|u|\right)$ is not necessary, since the exponential grows larger for $u>u_{l}$. Nonetheless, the chosen form in Eq. (2) allows one to clearly identify the confinement bounds.

[33] The SPDF was numerically obtained by taking uncorrelated samples after letting the system evolve for a long time $T$. The variability range of the output voltage $u$ was divided into $M$ cells, and the number of times $u$ took values within each cell was counted. The sampling interval $\Delta t$ was long enough so as to ensure independence.

[34] We point out that the theoretical SPDF curve displays maxima at 1 and -1 even when subjected to multiplicative noise, because this is simply additive for $|u|>\left|u_{l}\right|$. These are, however, masked by the fluctuations in the corresponding numerical curves.

[35] Despite the form of Eq. (5), $u=0$ remains to be an unstable fixed point.

[36] In the design of the Schmitt trigger device, an AD826 operational amplifier (whose input-output characteristic is centered around zero in order to simplify the analysis) was used as a comparator. Even though the AD826 is powered at $11 \mathrm{~V}$, an output of $\pm 5 \mathrm{~V}$ is obtained instead of the theoretical $\pm 5.5 \mathrm{~V}$, since the operational amplifier does not operate in the rail-to-rail regime.

[37] S. O. Rice, in Selected Papers on Noise and Stochastic Processes, edited by N. Wax (Dover, New York, 1954), pp. 189-195.

[38] The exponential amplifier is based on the natural $I_{d}-V_{d}$ input-output characteristic of rectifying diodes. This can be conceptually decomposed into two regimes: from 0 to $\sim 0.7 \mathrm{~V}$, the diode basically does not conduct; from $0.7 \mathrm{~V}$ on, the $I_{d}-V_{d}$ characteristic is actually of exponential form. With the goal of controlling the width of the well, the natural $0.7-\mathrm{V}$ threshold is varied in the circuit configuration, by inserting a variable dc voltage we call $u_{l}$. Also, the almost linear shape of the upper part of the wall is due to the fact that the diode characteristic is amplified with an AD826, in order to reach the amplifier's saturation as natural limits of the wall at 2 or $3 \mathrm{~V}$.

[39] Strictly, neither are the deterministic forces are fundamental: they are effective dissipative mesoscopic forces, so they are ultimately of entropic origin (they imply thermal baths that keep their parameters constant).

[40] V. V. Semenov, A. B. Neiman, T. E. Vadivasova, and V. S. Anishchenko, Phys. Rev. E 93, 052210 (2016).

[41] H. Risken, The Fokker-Planck Equation: Methods of Solution and Applications, 2nd ed. (Springer, Berlin, 1996). 